\title{
51. ON NONGRAVITATIONAL EFFECTS IN TWO CLASSES OF MODELS FOR COMETARY NUCLEI
}

\author{
O. V. DOBROVOL'SKIJ \\ Institute of Astrophysics, Dushanbe, U.S.S.R. \\ and \\ M. Z. MARKOVICH \\ Polytechnic Institute, Kalinin, U.S.S.R.
}

\begin{abstract}
Two types of cometary nuclei are considered: a homogeneous icy nucleus and an icy nucleus covered with a dispersive surface layer, $1 \mathrm{~cm}$ thick and having poor heat conductivity. The temperature of the evaporating ice surface of a nucleus rotating in a period of 6 hours about an axis perpendicular to the orbital plane was determined by numerical integration of the equation of heat conduction in the first case and of the Stefan problem in the second. For a nucleus of radius $10^{5} \mathrm{~cm}$ and mass of $3.8 \times 10^{15} \mathrm{~g}$ the reactive force was found to be about $10^{7} \mathrm{dyn}$ in either case, and the secular change in mean motion is of the order of the observed values. For the homogeneous nucleus the dependence of the reactive force on heliocentric distance is obtained.
\end{abstract}

In accordance with the ideas of Dubyago (1948) and Whipple (1950,1951), an evaluation is made of the order of magnitude of the reactive force $\boldsymbol{\Phi}_{r}$ and the corresponding nongravitational effect $\Delta n$ in the mean motion of a comet for two types of icy models for cometary nuclei.

\section{A Homogeneous Nucleus of $\mathrm{H}_{2} \mathrm{O}$ Ice}

In the case of a pure icy nucleus we take as the basis for our estimate of $\boldsymbol{\Phi}_{r}$ Meshcherskij's formula for the effect $\mathrm{d} \boldsymbol{\Phi}_{r}$ on an element $\mathrm{d} s$ of the nuclear surface

$$
\mathrm{d} \Phi_{r}=\frac{\mathrm{d} M}{\mathrm{~d} t} \mathbf{V} ; \quad \mathrm{d} \mathbf{w} \approx \frac{1}{M} \frac{\mathrm{d} M}{\mathrm{~d} t} \mathbf{V},
$$

where $\mathrm{d} M / \mathrm{d} t$ is the rate of escape of mass from the surface element, $w$ is the reactive acceleration, and $\mathbf{V}$ is the relative velocity of the escaping matter.

The nuclear temperature required for the determination of $\mathrm{d} M / \mathrm{d} t$ is found from the solution of the heat-conduction equation

$$
\frac{\partial T}{\partial t}=\frac{K}{\rho c} \frac{\partial^{2} T}{\partial x^{2}}
$$

where $T(x, t)$ is the nuclear temperature, $x$ the distance below the nuclear surface, $K$ the coefficient of thermal conductivity, $\rho$ the density, and $c$ the specific heat of the nuclear material.

The boundary condition is written as follows:

$$
q=-K(\operatorname{grad} T)_{x=0}+\varepsilon \sigma T^{4}+b,
$$


where $q$ is the heat received from the Sun, $\varepsilon$ is the degree of blackness of the surface, $\sigma$ the Stefan-Boltzmann constant, and $b$ the heat expended vaporizing the ice. For a nucleus with its axis of rotation perpendicular to its orbit plane

$$
q= \begin{cases}\frac{(1-A) q_{0}}{r^{2}} \sin \omega t \cos \varphi & 0 \leqslant \omega t \leqslant \pi \\ 0 & \pi \leqslant \omega t \leqslant 2 \pi\end{cases}
$$

where $q_{0}$ is the solar constant, $A$ the surface albedo, $r$ the heliocentric distance, $\omega t=\lambda$ is the longitude of the surface element (counted from the terminator), and $\varphi$ is the latitude of the surface element. The heat $b$ spent on the evaporation of the ice is given by

$$
b=L N
$$

where $L$ is the heat of evaporation of a molecule, and $N$ is the number of molecules evaporated from unit surface area per unit time:

$$
N=\frac{\alpha \mathscr{P}}{\sqrt{ }(2 \pi m k T)}
$$

with $\alpha$ being the evaporation coefficient, $\mathscr{P}$ the vapour pressure of saturated steam at the temperature $T, m$ the molecular mass, and $k$ Boltzmann's constant. According to the experiments on the evaporation of ice in a vacuum, $\alpha=1$ (Kajmakov and Sharkov, 1967). The initial condition takes the form

$$
T(x, 0)=f(x)
$$

The following values were adopted for the physical characteristics of $\mathrm{H}_{2} \mathrm{O}$ ice: $\rho=0.9 \mathrm{~g} \mathrm{~cm}^{-3}, \varepsilon=0.64, A=0.7, m=3 \times 10^{-23} \mathrm{~g}$ and $L=2 \times 10^{-20} \mathrm{cal}$; for $K$ and $c$ average values were taken for the range of temperatures $150-170 \mathrm{~K}$ from figures given by Shumskij (1955) and in Termicheskie Konstanty Neorganicheskikh Veshchestv, namely, $K=4.42 \times 10^{-3} \mathrm{cal} \mathrm{cm}^{-1} \mathrm{~s}^{-1} \mathrm{deg}^{-1}$, and $c=0.28 \mathrm{cal} \mathrm{g}^{-1} \mathrm{deg}^{-1}$. The pressure $\mathscr{P}$ in dyn $\mathrm{cm}^{-2}$ can be found from the semiempirical relation

$$
\log \mathscr{P}=-2720 / T+13.75
$$

Numerical integration of Equation (2) has been performed by the grid method (Markovich and Tulenkova, 1968) for a surface element at $\varphi=60^{\circ}$ and $r=1 \mathrm{AU}$, and with the initial condition $f(x)=155 \mathrm{~K}$. The period of nuclear rotation $P$ is taken as $6 \mathrm{~h}$. The influence of the initial conditions, which are always to some extent arbitrary, can be partly or totally eliminated by computing the temperature of the nucleus for several successive rotations. Figure 1 shows the nuclear temperature computed for the second rotation. The lag of the maximum surface temperature $T_{0}$ compared to the maximum of solar radiation is about $3 / 8 \mathrm{~h}\left(\frac{1}{16} P\right)$.

By integrating Equation (1) over the whole nuclear surface we may determine the acceleration components $S^{\prime}$ outward along the radius vector and $T^{\prime}$ perpendicular to 
it (in the orbit plane); $S^{\prime}$ and $T^{\prime}$ are determined (approximately) from the equations

$$
\begin{aligned}
& S^{\prime}=\frac{3}{2 \pi R_{0} \rho} \Delta \varphi \Delta \lambda \sum_{\varphi=0}^{\pi / 2} \sum_{\lambda=0}^{2 \pi} Q(\lambda, \varphi) v(\lambda, \varphi) \cos ^{2} \varphi \sin \lambda \\
& T^{\prime}=\frac{3}{2 \pi R_{0} \rho} \Delta \varphi \Delta \lambda \sum_{\varphi=0}^{\pi / 2} \sum_{\lambda=0}^{2 \pi} Q(\lambda, \varphi) v(\lambda, \varphi) \cos ^{2} \varphi \cos \lambda,
\end{aligned}
$$

where $R_{0}$ is the radius of the nucleus, $v$ is the average thermal velocity of molecules at the surface, and $Q=m N$ is the mass of matter escaping from unit surface area per unit time.

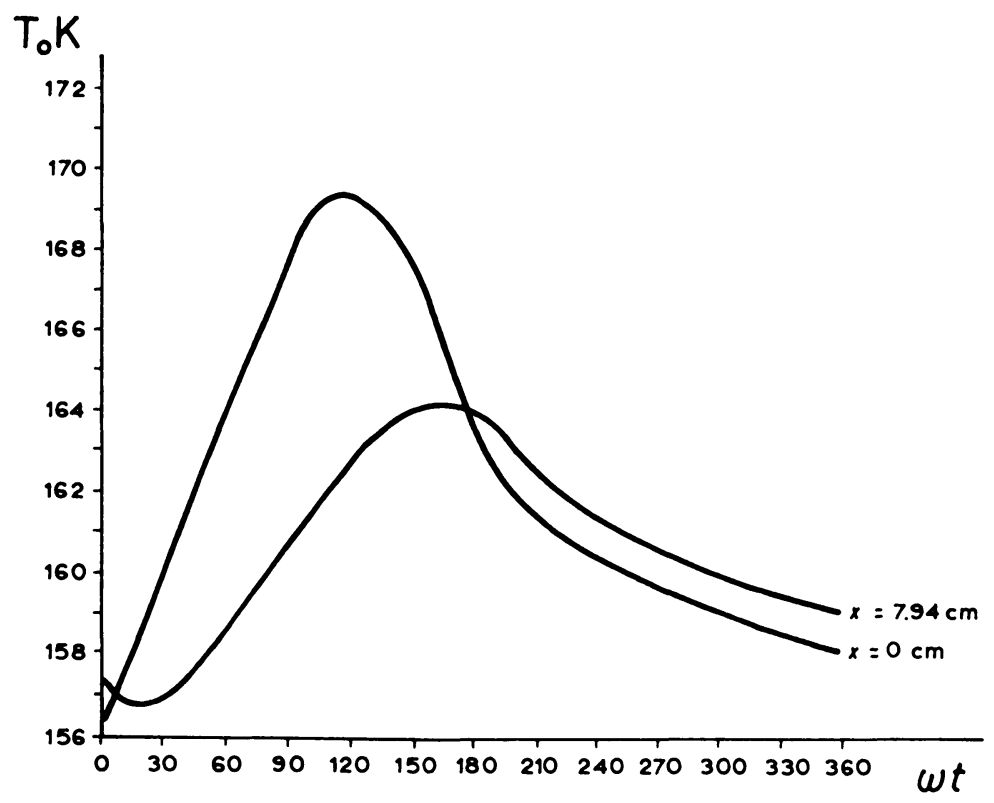

Fig. 1. Temperature variation $T_{0}$ on the surface and at a depth of $7.94 \mathrm{~cm}$ for a homogeneous icy nucleus of radius $1 \mathrm{~km}$ located at $1 \mathrm{AU}$ from the Sun and rotating with a 6 -h period.

For rough evaluation we took average values of $Q(\lambda, \varphi)$ and $v(\lambda, \varphi)$ for $\varphi=60^{\circ}$ from the temperature curve in Figure 1. For $R_{0}=10^{5} \mathrm{~cm}$ and $\rho=0.9 \mathrm{~g} \mathrm{~cm}^{-3}$ :

$$
\begin{aligned}
& S^{\prime}=1.4 \times 10^{-8} \mathrm{~cm} \mathrm{~s}^{-2} \\
& T^{\prime}=8.5 \times 10^{-9} \mathrm{~cm} \mathrm{~s}^{-2} \\
& \vartheta^{\prime}=\arctan \left(T^{\prime} / S^{\prime}\right)=31^{\circ} .
\end{aligned}
$$

The change in the mean motion $n$ for an orbit with semimajor axis $a$ and eccentricity $e$ is

$$
\frac{\mathrm{d} n}{\mathrm{~d} t}=-\frac{3 e \sin \theta}{a \sqrt{ }\left(1-e^{2}\right)} S^{\prime}-\frac{3 \sqrt{ }\left(1-e^{2}\right)}{r} T^{\prime}
$$


where $\theta$ is the true anomaly. Let us assume that $S^{\prime}$ changes symmetrically before and after perihelion. Then, in determining the average variation of $n$ per revolution, we need take only $T^{\prime}$ into account; the action of $S^{\prime}$ in this case will be only to diminish the effective gravitational constant. According to Equation (10), and taking $e=0$ and $r=1$ AU, we obtain $n=2.6 \times 10^{-6}$ arc sec per day or $10^{-3}$ arc sec per day per revolution, in order of magnitude agreement with observational data (Dubyago, 1948; Whipple, 1950, 1951; Marsden, 1968; Richter, 1963; Sekanina, 1967, 1968; Sitarski, 1964). It can easily be shown that $\Delta n$ will be of the same order for an elliptical orbit with semilatus rectum $1 \mathrm{AU}$.

For the nuclear model discussed the dependence of $w_{r}$ upon $r$ can be obtained. According to Whipple $(1950,1951)$,

$$
w_{r}=\zeta_{\eta} \frac{q_{0}}{r^{2}} v_{r}(\mathrm{eff})
$$

where $\zeta$ is a coefficient that depends on the properties of the nucleus (size, shape, etc.), and $\eta$ is the efficiency of evaporation (which depends on $r$ ). For $r>1 \mathrm{AU}$, $\eta=\eta_{0} r^{-k}$, and according to the computations by Markovich, $k=1.5$. For $r<1$ AU, $\eta$ fits very well the formula (Markovich and Tulenkova, 1968) $\eta=\left(1+r^{2.57}\right)^{-1}$.

Adopting the approximation $T_{0}=T_{00} r^{-\beta}(\beta \approx 0.2)$ for the temperature of the nuclear surface, we obtain $V_{r}$ (eff) $\sim \sqrt{T_{0}} \sim r^{-\beta / 2}$, whence

$$
\begin{array}{ll}
w_{r} \sim \frac{1}{r^{3.6}} & (r>1 \mathrm{AU}) \\
w_{r} \sim \frac{1}{r^{2.1}\left(1+r^{2.57}\right)} & (r<1 \mathrm{AU}) .
\end{array}
$$

\section{An Icy Nucleus with a Dusty Surface Layer}

Let us now discuss a nuclear model in the form of an ice $\left(\mathrm{H}_{2} \mathrm{O}\right)$ sphere of radius $R_{i}$, with a surface layer of thickness $l$ consisting of finely dispersive and poorly heat-conducting powder.

For determining the temperature of the evaporating ice surface directly beneath the dispersive layer, we shall make use of the so-called Stefan problem (Lykov, 1952; Tikhonov and Samarskij, 1966). Mathematically, this problem is formulated as follows:

$$
\begin{aligned}
& \frac{\partial T_{1}}{\partial t}=\frac{K_{1}}{\rho_{1} c_{1}} \frac{\partial^{2} T_{1}}{\partial x^{2}} \quad(0 \leqslant x \leqslant l) \\
& \frac{\partial T_{2}}{\partial t}=\frac{K_{2}}{\rho_{2} c_{2}} \frac{\partial^{2} T_{2}}{\partial x^{2}} \quad(x \geqslant l) \\
& T_{1}(x, 0)=f_{1}(x) \\
& T_{2}(x, 0)=f_{2}(x) \\
& T_{1}(l, t)=T_{2}(l, t)
\end{aligned}
$$




$$
\begin{aligned}
& q=-K_{1}\left(\operatorname{grad} T_{1}\right)_{x=0}+\varepsilon \sigma T^{4} \\
& b=-K_{1}\left(\frac{\partial T_{1}}{\partial x}\right)_{x=l}+K_{2}\left(\frac{\partial T_{2}}{\partial x}\right)_{x=l}=-L \rho_{2} \frac{\partial R_{i}}{\partial t} .
\end{aligned}
$$

The incident heat $q$ is determined from Equation (4), and the subscripts 1 and 2 refer to the surface layer and to the ice, respectively.

The flow of gas through the boundary of a capillary opening, with molecular flow taking place in the case of comets, is determined with the aid of the formula

$$
Q_{m}=\frac{2}{3} \frac{D}{l^{\prime}}\left(p_{2}-p_{1}\right) \sqrt{ }\left(\frac{2}{\pi} \frac{\mu}{R T}\right)
$$

where $D$ is the diameter of the capillary, $l^{\prime}$ its length, $\mu$ the molecular weight of the gas, $R$ is the universal gas constant, $T$ the temperature of the gas, and $\Delta p=p_{2}-p_{1}$ is the pressure difference at the ends of the capillary, $p_{1}$ being close to zero.

In calculating the flow of gas through porous materials, the corresponding effective values of $l^{\prime}$ and $D$ are taken to be (Lejbenson, 1934, 1947)

$$
\begin{aligned}
l^{\prime} & =l \sqrt{ }\left[\frac{6}{\pi}\left(1-p^{\prime}\right)\right] \\
D & =0.52 R_{g},
\end{aligned}
$$

where $p^{\prime}$ is the porosity, and $R_{g}$ is the radius of the grains of dispersive material.

Heat spent for the sublimation of ice is determined from the relation

$$
b=\left(\mathscr{P}-p_{2}\right)(2 \pi m k T)^{-1 / 2},
$$

with $\mathscr{P}$ the pressure of saturated steam, determined from Equation (8), and $p_{2}$ the actual gas pressure in the pores of the surface layer directly adjoining the internal icy nucleus. Hence

$$
p_{2}=\mathscr{P}-\frac{b}{L}(2 \pi m k T)^{1 / 2}
$$

where $b$ is found from Equation (17).

The problem is solved by numerical integration for a surface element on the equator of the nucleus ( $\varphi=0, r=1 \mathrm{AU})$, the period $P$ being taken as $6 \mathrm{~h}$ and the rotation axis assumed perpendicular to the plane of orbit. The formulae by Markovich and Tulenkova (1968) are used for the purpose.

The temperature of the evaporating ice surface (layer $x=l$ ) is determined by interpolation from the temperatures of four layers equidistant from the surface, two in the ice region and two in the surface layer.

The components of the reactive force are calculated from the relations:

$$
\begin{aligned}
& S^{\prime \prime}=\frac{3 p^{\prime}}{2 \pi R_{0} \rho} \Delta \varphi \Delta \lambda \sum_{\varphi=0}^{\pi / 2} \sum_{\lambda=0}^{2 \pi} Q_{m}(\lambda, \varphi) V_{r}(\lambda, \varphi) \cos ^{2} \varphi \sin \lambda \\
& T^{\prime \prime}=\frac{3 p^{\prime}}{2 \pi R_{0} \rho} \Delta \varphi \Delta \lambda \sum_{\varphi=0}^{\pi / 2} \sum_{\lambda=0}^{2 \pi} Q_{m}(\lambda, \varphi) V_{r}(\lambda, \varphi) \cos ^{2} \varphi \cos \lambda,
\end{aligned}
$$


where $p^{\prime}$ is the area of the capillary openings as a fraction of the total nuclear surface, and it is taken to be equal to the porosity of the surface layer material. To simplify the calculations $Q_{m}(\lambda, \varphi)$ is replaced, as earlier, by the average value $\frac{1}{2} Q_{m}(\lambda, 0)$.

The following physical characteristics of the surface layer of the nucleus have been adopted: $A=0.07, l=1 \mathrm{~cm}, R_{g}=5 \times 10^{-4} \mathrm{~cm}, p^{\prime}=0.80, \rho_{1}=0.8 \mathrm{~g} \mathrm{~cm}^{-3}, c_{1}=0.2 \mathrm{cal}$ $\mathrm{g}^{-1} \mathrm{deg}^{-1}, K_{1}=3 \times 10^{-6} \mathrm{cal} \mathrm{cm}^{-1} \mathrm{~s}^{-1} \mathrm{deg}^{-1}$. Heat exchange by radiation can be neglected in this case, for according to Reprintseva and Fedorovich (1968), the influence of temperature on heat radiation is important only in systems consisting of particles exceeding $1 \mathrm{~mm}$ in size; starting at $10{ }^{\circ} \mathrm{C}$ the role of radiation in such a system increases, and it reaches quite a significant amount at $100^{\circ} \mathrm{C}$. For the ice we take $c_{2}=0.36 \mathrm{cal} \mathrm{g}^{-1} \mathrm{deg}^{-1}$ and $K_{2}=4.7 \times 10^{-3} \mathrm{cal} \mathrm{cm}^{-1} \mathrm{~s}^{-1} \mathrm{deg}^{-1}$ as average values for the temperature range 150-200 K, the other characteristics being the same as in Section 1.

Figure 2 shows examples of temperature curves calculated for this model. The

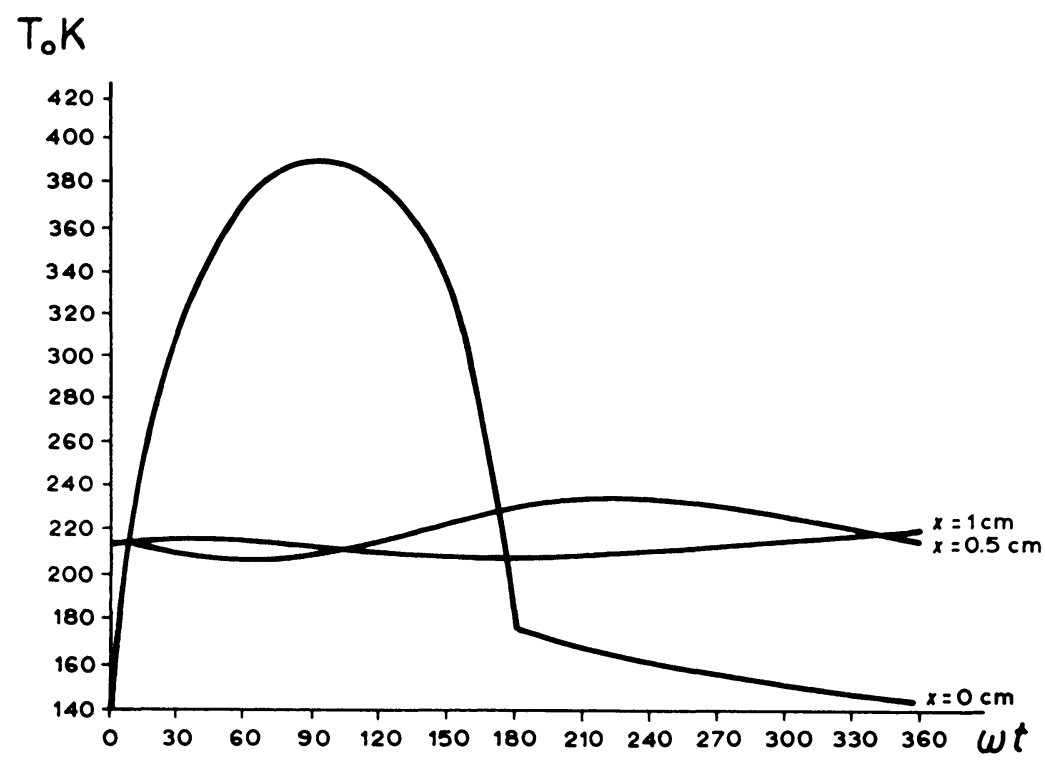

Fig. 2. Temperature variation $T_{0}$ on the surface and at depths of 0.5 and $1.0 \mathrm{~cm}$ for an icy nucleus covered by a $1 \mathrm{~cm}$ dust layer.

temperature $T_{0}$ of the nuclear surface $(x=0)$ on the illuminated side essentially follows the variation in solar radiation. The temperature of the evaporating ice surface does not depend on $T_{0}$ : in our case it shows small variations which are not synchronous with the rotation of the nucleus. It is thus possible that the average value of the reactive acceleration will be close to zero.

For the particular case shown in Figure 2, with $R_{0}=10^{5} \mathrm{~cm}$ and $\rho=0.9 \mathrm{~g} \mathrm{~cm}^{-3}$, it follows for Equation (22) that 


$$
\begin{aligned}
& S^{\prime \prime}=7 \times 10^{-9} \mathrm{~cm} \mathrm{~s}^{-2} \\
& T^{\prime \prime}=9 \times 10^{-9} \mathrm{~cm} \mathrm{~s}^{-2} \\
& \vartheta^{\prime \prime}=52^{\circ} .
\end{aligned}
$$

Hence, for a circular orbit with $r=1 \mathrm{AU}$, from Equation (11) we have again $\Delta n=10^{-3}$ arc sec per day per revolution.

Thus the icy model, which is widely accepted and agrees. well with photometric data, automatically leads to nongravitational effects in the motions of comets, and they are largest near perihelion. It is also quite possible that nuclei coated with dust would produce nongravitational effects.

\section{References}

Dubyago, A. D.: 1948, Astron. Zh. 20, 361.

Kajmakov, E. A. and Sharkov, V. I.: 1967, Komety $i$ Meteory No. 15, 16.

Lejbenson, L. S.: 1934: Neftepromyslovaya Mekhanika, Gorgeolnefteizdat, Moscow, Groznyj, Leningrad and Novosibirsk, part 2.

Lejbenson, L. S.: 1947, Dvizhenie Prirodnykh Zidkostej i Gazov v Poristoj Srede, Gostekhizdat, Moscow-Leningrad.

Lykov, A. V.: 1952, Teoriya Teploprovodnosti, Gostekhizdat, Moscow.

Markovich, M. Z. and Tulenkova, L. N.: 1968, Probl. Kosmich. Fiz. No. 3, 25.

Marsden, B. G.: 1968, Astron. J. 73, 367.

Reprintseva, S. M. and Fedorovich, N. V.: 1968, Luchistyj Teploobmen v Dispersnykh Sredakh, Nauka i Tekhnika, Minsk.

Richter, N. B.: 1963, The Nature of Comets, Methuen, London.

Sekanina, Z.: 1967, Bull. Astron. Inst. Czech. 18, 15.

Sekanina, Z.: 1968, Bull. Astron. Inst. Czech. 19, 210.

Sitarski, G.: 1964, Acta Astron. 14, 323.

Shumskij, P. A.: 1955, Osnovy Structurnogo Ledovedeniya, AN SSSR, Moscow.

Tikhonov, A. N. and Samarskij, A. A.: 1966, Uravneniya Matematicheskoj Fiziki, Nauka, Moscow.

Whipple, F. L.: 1950, Astrophys. J. 111, 375.

Whipple, F. L.: 1951, Astrophys. J. 113, 464. 